\title{
Selection of subcontractors using ordinal ranking methods based on Condorcet approach
}

\author{
Sławomir Biruk, Piotr Jaśkowski \\ Department of Construction Methods and Management, \\ Faculty of Civil Engineering and Architecture, Lublin University of Technology, \\ e-mail: s.biruk@pollub.pl,p.jaskowski@pollub.pl
}

\begin{abstract}
A choice of a subcontractor may have critical impact on realization of the project, it has influence on the cost, duration, and quality. Selection of the best sucontractor can be defined as multiple criteria decision making problem (MCDM) of choosing a proper offer from set of alternatives evaluated by using set of criteria. Decision maker should determine the criteria as objective and measurable. Significance of decision making problem is presented by large amount of theories and methods developed for solving MCDM problems and number of criteria considered in these problems. A Condorcet method (formulated over two centuries ago) is commonly accepted for democratic (majority of criteria determines the winner) and fair election - a Condorcet winner is the alternative which is preferred in all pair-wise comparisons. According to social choice theory where a Condorcet winner cannot be obtained from a set of alternatives, the best solution is close to being a Condorcet winner. The paper presents four selection methods of the best alternative that is as close as possible to being a Condorcet winner and contains examples of a subcontractor selection using only ordinal scales of evaluation of alternatives.
\end{abstract}

Keywords: project management, subcontractors selection, social choice theory, the Condorcet winner.

\section{Multi criteria subcontractor selection problem}

A general contractor evaluates bid proposals for a construction works taking into account several criteria, for example costs, project duration or contractor's experience. He must make the best compromise choice among the set of alternatives (subcontractors) $A=\left\{a_{1}, \ldots, a_{n}\right\}$ evaluated using set of criteria $C=\left\{c_{1}, \ldots, c_{n}\right\}$. The importance of the criteria is usually different from point of view of the general contractor, therefore often criteria have assigned weights. Variants evaluation should be performed to fulfill decision maker requirements. The criteria should be the most objective and measurable. In the case of using many criteria, the objective evaluation of variants is difficult, especially that part of criteria is quantitative and part is qualitative. That problem can be effectively solved only by application of objective multi-attribute decision making methods. They allow to apply many criteria, also contradictory to each other. Variety of applied methods and procedures to aid decision making testifies about problem complexity and about difficulty in formulation of universal solutions. The method should be appropriate to solve multi faced problem.

Factors influence on subcontractors selection were identified by many researches by questionnaire survey $[1,2,3]$. Many of them are qualitative (e.g. safety and health at work, 
instruction and training or reputation) so the popular method of subcontractors selection is Analytic Hierarchy Process $(A H P)[4,5,6,7]$. This is because the decision maker must only define relative dominance of alternatives. In case of qualitative criteria, pairwise comparison of relative dominance of alternatives replaces necessity of quantification of alternatives ratings. Number of elements compared should be small (not more than 9) to improve accuracy of measurement [8].

The information obtained about the subcontractors are often approximate, imprecise and their scope is insufficient to make a decision. The decision-maker in many cases can easier determine relative preferences by identifying the range of values or in narrative form rather than by giving specific values form the accepted range of grades. Fuzzy set theory allows to incorporate uncertainty measures to multi attribute decision $[9,10]$.

Arrow and Raynauld [11] presented an analogy between social choice theory and multi-criteria decision-making methods. A rank in criterion order can be treated as electoral votes. In ordinal ranking methods there is no need to evaluate alternatives against criteria using interval or ratio scale in case quantitative criteria and convert qualitative into quantitative. There is no need to determine weights of criteria too. For all criteria a decision maker creates a preference relation (linear order) of variants. For each criterion variants are numbered in increasing order from 1 to $n$ according to a decision maker preference. Preferred variant $r$ to $s$ respect to criterion $j$ occurs before $s$ in profile $j$ and his rank is smaller then $s$. Pairwise comparision of variants allows to create an outraking matrix $\mathbf{W}$. Entries $w(r, s)$ are number of criteria where $r$ is prefered to $s$. Winning margin $u(r, s)$ is defined as the difference between the number of criteria preferring $r$ variant relative to $s$, so $\mathbf{U}=\mathbf{W}-\mathbf{W}^{\mathrm{T}}$. Based on preference relations for each criterion it is possible to identify the winner or create collective preference order.

\section{Subcontractor selection using condorcet method}

Condorcet more than two centuries ago formulated a rule, that winner is a variant $r$ such $\forall s \neq r, w(r, s)>m / 2$ (a Condorcet winner defeats each variant in more than $50 \%$ of criteria) or $u(r, s)>0$. Condorcet looser defeats in pairwise comparison with all others. Linear order satisfied Condorcet's rule, if preferred variant $r$ to $s$ occurs before $s$ (the rank $r$ is smallest than $s$ ) for all $r \neq s$. Condorcet's winner, looser and order not always exist in a given set of criteria. A Condorcet method (formulated over two centuries ago) is commonly accepted for democratic (majority of criteria determines the winner) and fair election - a Condorcet winner is the alternative which is preferred in all pair-wise comparisons.

Pairwise comparison results can be presented as a complete asymmetric directed graph $T=(A, E)$ so called tournament, which consists of a set of vertex (variants) $A(T)$ and set of $\operatorname{arcs} E(T) \subseteq A(T) \times A(T)$. The arc $(r, s)$ exists if variant $r$ is preferred to $s$ $(r \neq s)$ by majority of criterion. The tournament $T$ is transitive if $(i, j) \in T$ and $(j, k) \in T$ implies $(i, k) \in T$. There exists overall linear order which complies with a preference relation for each criterion in transitive tournament.

In case when it is not possible to select the Condorcet winner from the social choice theory point of view, the chosen candidate should be ,as closely as possible” to Condorcet winner. Approximate algorithm to choose the winner should satisfy Condorcet criterion and place a Condorcet winner in the first place if it exists. The algorithm should introduce minimal perturbances is preference relations for criteria when variant is turn to a Condorcet winner. 
Finding Slater solution [12] is based on determining the minimal number of arc in tournament $T=(A, E)$ which should be reversed to determine linear orders, what is equivalent with selection of a Condorcet winner. A Slater order minimizes distance between overall ranking and a preference relation for criteria according to the majority rule. A few Slater orders can exist, but in each Condorcet Winner is a Slater Winner [13].

Computation Slater order is $N P$-hard equivalent to the minimum feedback arc set problem on tournaments $[14,15]$. A Slater order can be find by solving integer linear program [16]:

$$
\begin{aligned}
& \max : \sum_{(i, j) \in E} w_{i j} x_{i j} \\
& x_{i j}+x_{j k}+x_{k i} \leq 2, \forall i, j, k \\
& x_{i j}+x_{j i}=1, \quad \forall i, j(i \neq j) \\
& x_{i j}=\{0,1\}, \forall i, j
\end{aligned}
$$

Weight of arc $w(i, j)=1$ if and only if $(i, j) \in T,-1$ otherwise. Binary variable $x_{i j}=1$ when $(i, j) \in T$ and takes value 0 otherwise, so the goal function (1) maximizes number of arcs used from the tournament to transform him into a linear order (minimizing number of reversing arcs in tournament $T$ ). Condition (2) is a transitive constrain (eliminates directed cycles of length 3 in the tournament), set of constraints (3) and (4) cause that only one directed arc joining two different nodes exists.

Young score [17] for variant $r$ is maximal subset of profiles (or the minimal number of profiles have to be ignored) which should be taken into account to determine a Condorcet winner). The maximal number of profiles is called Young score. The Young winner is a variant with the highest Young score. Approximate the Young score is NP-hard [18, 19] .

Let $a_{r} \in A$ be a variant for which Young score is calculated, $x_{j} \in\{0,1\}$ binary variable. Variable $x_{j}=1$ if criterion $j$ belongs to the subset of profiles which are included for computing Young's score for $a_{r}$. Constant $e_{r j}^{i}$ depends on preference relations for criteria and $e_{r j}^{i}=1(r \neq j)$ when in profile $j$ variant $a_{r}$ is preferred than $a_{i}$ or $e_{r j}^{i}=-1$ when in profile $j$ variant $a_{i}$ is preferred than $a_{r}$. Young score for $a_{r}$ can be obtained by solving integer linear program [19]:

$$
\begin{aligned}
& \max : \sum_{j=1}^{m} x_{j} \\
& \sum_{j=1}^{m} x_{j} \cdot e_{r j}^{i} \geq 1, \forall a_{i} \in A \backslash\left\{a_{r}\right\} \\
& x_{j}=\{0,1\}, j=1,2, \ldots m
\end{aligned}
$$

The goal function (5) maximizes number of profiles which cause that variant $a_{r}$ is turned into the Condorcet winner - equation (6).

Moving variant $a_{r}$ in individual ranking for criterion $j$ by $k\left(k \in\{0,1, \ldots, n-1\}\right.$ position causes that winning margin for $a_{r}$ over $a_{i} \in A \backslash\left\{a_{r}\right\}$ increases by $e_{i j k}=\{0,1\}$. Constant $d_{i}\left(a_{r}\right)$ is minimal number of criteria in which $a_{r}$ additionally has to get additional vote to defeat $a_{i} \in A \backslash\left\{a_{r}\right\}$ (the deficit of criterion votes 
for variant $a_{r}$ ). If $a_{r}$ is preferred in relation to $a_{i} \in A \backslash\left\{a_{r}\right\}$ then $d_{i}\left(a_{r}\right)=0$. The least number of switches in rankings for criteria is called Dodgston score $D\left(a_{i}\right)$. Dodgsone is more often known as Lewis Carroll. Computing a Dodgson winner is $N P$-hard problem [20, 21]. Dodgson's winner is a variant with the smallest value $D\left(a_{i}\right)$. For variant $a_{r}$ Dogstone score can be computed by solving integer linear program [20]:

$$
\begin{aligned}
& \min : \sum_{j} \sum_{k} k \cdot x_{j k} \\
& \sum_{k} x_{j k}=1, \forall j \\
& \sum_{j} \sum_{k} e_{i j k} \cdot x_{j k} \geq d_{i}\left(a_{r}\right), \forall a_{i} \in A \backslash\left\{\mathrm{a}_{\mathrm{r}}\right\} \\
& x_{j k}=\{0,1\}, \forall j, k
\end{aligned}
$$

Binary variable $x_{j k}=1$ when in preference order type $j$ variant $a_{r}$ is moved upwards by $k$ positions. The goal function (8) minimizes number of switches in preference orders for criteria, set of constraints (9) cause that in each preference order it is possible to move $a_{r}$ only one time. Set of constraints (10) ensures that $a_{r}$ variant becomes the Condorcet winner.

In Condorcet least-reversal system a winner is the variant which became the Condorcet after reversing the minimum possible number of pairwise comparisons [22]. After making the minimum number of reversals a new outranking matrix is created with entries $w_{c l v}^{r}(r, s)$. For variant $a_{r}$ the distance between two profiles origin and after revising can be calculated as follows:

$$
d_{r}=\frac{1}{2} \sum_{r, s \in A}\left|w(r, s)-w_{c l v}^{r}(r, s)\right|
$$

The winner is a variant with minimal distance. In a simpler way the winner is the variant $a_{r}$ minimizing [23]:

$$
\left.l_{r}=\sum_{s=1}^{n} n e g(u(r, s))\right), \forall a_{s} \in A \backslash\left\{\mathrm{a}_{\mathrm{r}}\right\}
$$

where:

$$
n e g(x)=\left\{\begin{array}{ccc}
-x & \text { if } & x \leq 0 \\
x & \text { if } & x>0
\end{array}\right.
$$

\section{A case study of subcontractor selecting using the Condorcet rule}

Application of the Condorcet method to select te subcontractor will be presented on the example of selection one of the five offers evaluated using five criteria: cost (1), duration time (2), quality (3), subcontractor experience (4) and warranty (5). Decision maker prepared ranking of subcontractors for each criterion as follows: 


$$
\begin{aligned}
& c_{1}: a_{4} \succ a_{2} \succ a_{5} \succ a_{1} \succ a_{3}, \\
& c_{2}: a_{3} \succ a_{5} \succ a_{2} \succ a_{1} \succ a_{4}, \\
& c_{3}: a_{2} \succ a_{1} \succ a_{4} \succ a_{3} \succ a_{5} \\
& c_{4}: a_{3} \succ a_{5} \succ a_{1} \succ a_{2} \succ a_{4} \\
& c_{5}: a_{1} \succ a_{3} \succ a_{2} \succ a_{5} \succ a_{4},
\end{aligned}
$$

In this example there is no a Condorcet Winner, because the offer which beats others in pairwise comparisons do not exist. In the tournament there are two directed cycles: 1-3-21 and 1-3-5-1. Variant $a_{5}$ is the Condorcet looser. The pairwise comparison graph of example is shown in Fig. 1.

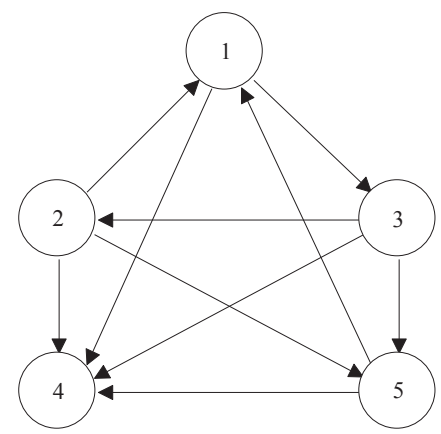

Fig. 1. The majority tournament of an example

By reversing only one arc $(1,3)$ we obtain unique Slater order (3-2-5-1-4) and offer $a_{3}$ became the Slater winner (Figure 2).

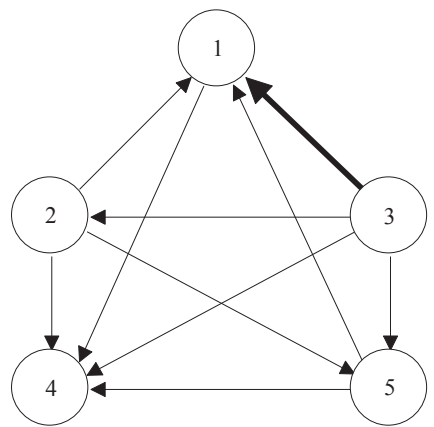

Fig. 2. The Slater acyclic tournament for the example

Ignoring the preference orders for criteria $c_{1}$ and $c_{2}$ we return offer $a_{1}$ into the Condorcet winner:

$$
\begin{aligned}
& c_{3}: a_{2} \succ a_{1} \succ a_{4} \succ a_{3} \succ a_{5} \\
& c_{4}: a_{3} \succ a_{5} \succ a_{1} \succ a_{2} \succ a_{4} \\
& c_{5}: a_{1} \succ a_{3} \succ a_{2} \succ a_{5} \succ a_{4},
\end{aligned}
$$

and Young score for $a_{1}$ is equal 3. 
For offer $a_{2}$ we must include profiles for criteria $c_{1}, c_{2}$ and $c_{3}$ :

$$
\begin{aligned}
& c_{1}: a_{4} \succ a_{2} \succ a_{5} \succ a_{1} \succ a_{3}, \\
& c_{2}: a_{3} \succ a_{5} \succ a_{2} \succ a_{1} \succ a_{4}, \\
& c_{3}: a_{2} \succ a_{1} \succ a_{4} \succ a_{3} \succ a_{5}
\end{aligned}
$$

so $\mathrm{Y}\left(a_{2}\right)=3$.

Similarly $\mathrm{Y}\left(a_{3}\right)=3\left(c_{2}, c_{4}\right.$ and $c_{5}$ criteria are included $), \mathrm{Y}\left(a_{4}\right)=1-c_{1}$ criteriin. The offer $a_{5}$ cannot be a Condorcet winner. Consequently the overall preference order according to Young score is following: $a_{1} \approx a_{2} \approx a_{3} \succ a_{4} \succ a_{5}$.

The $a_{1}$ offer loses with $a_{2}$ and $a_{5}$ in relation 2:3. According to the Dodgson's rule, to make $a_{1}$ the Condorcet winner, it's enough to place the solution in ranking for $c_{1}$ in the second position:

$$
\begin{aligned}
& c_{1}: a_{4} \succ a_{1} \succ a_{2} \succ a_{5} \succ a_{3}, \\
& c_{2}: a_{3} \succ a_{5} \succ a_{2} \succ a_{1} \succ a_{4}, \\
& c_{3}: a_{2} \succ a_{1} \succ a_{4} \succ a_{3} \succ a_{5} \\
& c_{4}: a_{3} \succ a_{5} \succ a_{1} \succ a_{2} \succ a_{4} \\
& c_{5}: a_{1} \succ a_{3} \succ a_{2} \succ a_{5} \succ a_{4},
\end{aligned}
$$

or for $c_{2}$ move upwards at two positions:

$$
c_{2}: a_{3} \succ a_{1} \succ a_{5} \succ a_{2} \succ a_{4} \text {. }
$$

Hence Dodgston's score is equal $D\left(a_{1}\right)=2$. The same results can be obtain by moving $a_{1}$ at one position in ballots for criteria $c_{1}$ and $c_{2}$ or for $c_{3}$ and $c_{4}$.

The offer $a_{2}$ loses only with $a_{3}$ in relation 2:3. Moving in ranking for $c_{5}$ criterion at one position:

$$
\begin{aligned}
& c_{1}: a_{4} \succ a_{2} \succ a_{5} \succ a_{1} \succ a_{3}, \\
& c_{2}: a_{3} \succ a_{5} \succ a_{2} \succ a_{1} \succ a_{4}, \\
& c_{3}: a_{2} \succ a_{1} \succ a_{4} \succ a_{3} \succ a_{5} \\
& c_{4}: a_{3} \succ a_{5} \succ a_{1} \succ a_{2} \succ a_{4} \\
& c_{5}: a_{1} \succ a_{2} \succ a_{3} \succ a_{5} \succ a_{4},
\end{aligned}
$$

causes that $a_{2}$ becomes the Condorcet winner $-D\left(a_{2}\right)=1$.

Similarly: $D\left(a_{3}\right)=1$ by changing the position of $a_{3}$ and $a_{1}$ in profile for criterion $c_{1}$ or $c_{5}$. The offer $a_{4}$ needs two additional votes against $a_{1}$ and $a_{2}$ and one against $a_{3}$ and $a_{5}$. Changing for example preferences of orders for $c_{2}: a_{4} \succ a_{3} \succ a_{5} \succ a_{2} \succ a_{1}$ and $c_{4}: a_{3} \succ a_{5} \succ a_{4} \succ a_{1} \succ a_{2}$ we get Dodgson's score $D\left(a_{4}\right)=6$. Moving $a_{5}$ at one position in preferences of orders for $c_{1}, c_{2}$ and $c_{3}$ :

$$
\begin{aligned}
& c_{1}: a_{4} \succ a_{5} \succ a_{2} \succ a_{1} \succ a_{3}, \\
& c_{2}: a_{5} \succ a_{3} \succ a_{2} \succ a_{1} \succ a_{4}, \\
& c_{3}: a_{2} \succ a_{1} \succ a_{4} \succ a_{5} \succ a_{3}
\end{aligned}
$$

we have $D\left(a_{5}\right)=3$. 
Overall ranking in Dodgon method is following: $a_{2} \approx a_{3} \succ a_{1} \approx a_{5} \succ a_{4}$.

In Condorcet least-reversal system outranking matrices $\mathbf{W}, \mathbf{U}$ are created and next sums of rows are calculated according to (13) and (14) as follows:

$$
\mathbf{W}=\left[\begin{array}{ccccc}
- & 2 & 3 & 4 & 2 \\
3 & - & 2 & 4 & 3 \\
2 & 3 & - & 3 & 4 \\
1 & 1 & 2 & - & 2 \\
3 & 2 & 1 & 3 & -
\end{array}\right], \mathbf{U}=\left[\begin{array}{ccccc}
- & 1 & 0 & 0 & 1 \\
0 & - & 1 & 0 & 0 \\
1 & 0 & - & 0 & 0 \\
3 & 3 & 1 & - & 1 \\
0 & 1 & 3 & 0 & -
\end{array}\right], \mathbf{L}=\left[\begin{array}{c}
2 \\
1 \\
1 \\
8 \\
4
\end{array}\right]
$$

and as a consequence: $a_{2} \approx a_{3} \succ a_{1} \succ a_{5} \succ a_{4}$.

The summery results are shown in Table 1 .

Table 1. Overall preference order for applied methods

\begin{tabular}{|l|c|c|c|c|c|}
\hline \multirow{2}{*}{\multicolumn{1}{c|}{ Method }} & \multicolumn{6}{c|}{ Rank of subcontractor } \\
\cline { 2 - 6 } & $a_{1}$ & $a_{2}$ & $a_{3}$ & $a_{4}$ & $a_{5}$ \\
\hline Slater's rule & 4 & 2 & 1 & 5 & 3 \\
\hline Young's rule & 1 & 1 & 1 & 4 & 5 \\
\hline Dodgson's rule & 3 & 1 & 1 & 5 & 4 \\
\hline Condorcet least-reversal system & 3 & 1 & 1 & 5 & 4 \\
\hline
\end{tabular}

\section{Conclusions}

A choice of subcontractor impacts on a construction project duration, cost and quality. Selection of the best subcontractor can be defined as MCDM problem of choosing a proper offer from the set of alternatives evaluated by using the set of criteria. Social choice theory can be also applied in a supplier selection, who is evaluated using many criteria, where the position in criteria ranking is identified with the voices of voters. The offers evaluation requies only to prepare a preference relation for each criteria what simplifies decision making in the case of using many incommensurable criteria. The article presents ranking rules: Slater, Young, Dogstone and Condorcet least-reversal system. The application of those methods allows to chose the candidate closest to the Condorcet winner, in case whten Condorcet winner do not exist. The way of choosing the best offer is intuitive and commonly understood. The difference is in computational complexity and may lead to different overall orders arrangements, but place the Condorcet Winner on the first place if it exists. Author to solve the integer linear programs to determine the winner used LINGO 14.0 Optimization Modeling Software. Implementation of ranking methods to choose the offers in construction requires to elaborate easy to use computer software, what will be the further stage of author works.

\section{Acknowledgments}

This work was financially supported by Ministry of Science and Higher Education in Poland within the statutory research number S/63/2016. 


\section{References}

1. Marzouk M.M., El Kherbawy A.A. and Khalifa M. Factors Influencing Sub-contractors Selection in Construction Projects. HBRC Journal 9 (2013) 150-158.

2. Ng S.T., Skitmore M. and Chung,W.F. Ten Basic Factors to Indentify Suitable Subcontractors for Construction Projects. Proc. CIB TG 23 International Conference, Hong Kong, 2003.

3. Taruna D., Rajiv Bhatt R. and Bhavsar J.J. Methodology for Ranking of Factors Affecting Selection of Subcontractor for Construction Contractors of Gujarat. International Journal of Engineering Trends and Technology (IJETT) 32(1) (2016) 19-25.

4. Al-Harbi K.M.A.-S. Application of the AHP in Project Management. International Journal of Project Management 19(1) (2001) 19-27.

5. Alptekin O. Multi-Criteria Decision Making Approach in Contractor Selection. International Journal of Natural and Engineering Sciences 8(2) (2014) 6-9.

6. Anagnostopoulos K.P., Vavatsikos A.P. An AHP Model for Construction Contractor Prequalification. Operational Research. An International Journal 6(3) (2006) 333-346.

7. Fong P.S.-W., Choi S.K.-Y. Final Contractor Selection Using the Analytical Hierarchy Process. Construction Management and Economics 18 (2000) 547-557.

8. Saaty R.W. The Analytic Hierarchy Process - What It Is and How It Is Used. Mathematical Modelling 9(3-5) (1987) 161-176.

9. Mohaghar A., Faqhei M.S. Contractor Selection Using Extended TOPSIS Technique with Interval - Valued Triangular Fuzzy Numbers. Global Business and Economics Research Journal 2(5) (2013) 55-65.

10. Plebankiewicz E. A Fuzzy Sets Based Contractor Prequalification Procedure. Automation in Construction 22 (2012) 433-443.

11. Arrow K.J., Raynaud H. Social Choice and Multicriterion Decision Making. M.I.T. Press, Cambridge, 1986.

12. Slater P. Inconsistencies in a Schedule of Paired Comparisons. Biometrika 48 (1961) 303-312.

13. Hudry O. On the Difficulty of Computing the Winners of a Tournament. Annales du LAMSADE n 6, actes du Workshop on Voting Theory and Preference Modelling, DIMACS (2006) 181-191.

14. Charon I., Hudry O. A branch-and-Bound Algorithm to Solve the Linear Ordering Problem for Weighted Tournaments. Discrete Applied Mathematics 154 (2006) 2097-2116.

15. Festa P., Pardalos P.M., Resende M.G.C. Feedback Set Problems, [in:] Handbook of Combinatorial Optimization (Eds. Du D.Z., Pardalos P.M.). Vol. A, Kluwer 2009, 209-258.

16. Sukegawa N., Mizuno S. Redundancy of the Transitivity Constraints in the Ordering Problem. Department of Industrial Engineering and Management, Technical Report No. 2014-2, Tokyo Institute of Technology JAPAN, 2104.

17. Young H.P. Extending Condorcet's Rule. Journal of Economic Theory 16 (1997) 335-353.

18. Betzler N., Guo J., Niedermeier R. Parameterized Computational Complexity of Dodgson and Young Elections. Information and Computation 208(2) (2010) 165-177.

19. Caragiannis I., Cove, J.A., Feldma, M., Homan C.M., Kaklamanis C., Karanikolas N., Procaccia A.D., Rosenschein J.S. On the Approximability of Dodgson and Young Elections. Artificial Intelligence (187-188) (2012) 31-51.

20. Bartholdi III J., Tovey C.A., Trick M.A. Voting Schemes for Which It Can Be Difficult to Tell Who Won the Election. Social Choice and Welfare 6(2) (1989) 157-165.

21. Tideman T.N. Independence of Clones as a Criterion for Voting Rules. Social Choice and Welfare 4(3) (1987) 185- 206.

22. Nurmi H. Settings of Consensual Processes: Candidates, Verdicts, Policies, [in:] Consensual Processes. Studies in Fuzziness and Soft Computing. (Eds. Herrera-Viedma E., García-Lapresta J.L., Kacprzyk J., Fedrizzi M., Nurmi H. and Zadrożny S.), Berlin Heidelberg, Springer, 2011, 159-178.

23. Smith W.D. Descriptions of Single-Winner Voting Systems. temple.edu/wds/ homepage/votedesc.pdf, 2006. 